\title{
IgG Antibody Response to Polyethylene Glycol-modified Adenosine Deaminase in Patients with Adenosine Deaminase Deficiency
}

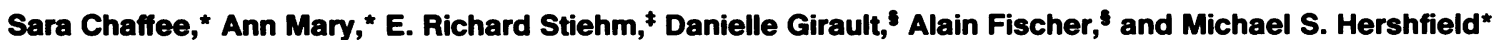 \\ ${ }^{*}$ Departments of Medicine, Biochemistry, and Pediatrics, Duke University Medical Center, Durham, North Carolina $27710 ;{ }^{\ddagger}$ Department \\ of Pediatrics, University of California Los Angeles, Los Angeles, California; and ${ }^{\S}$ Department of Pediatrics, Hôpital Necker, Paris, France
}

\begin{abstract}
Polyethylene glycol (PEG)-modified bovine adenosine deaminase (ADA) is used for replacement therapy of severe combined immunodeficiency disease due to inherited ADA deficiency. We monitored IgG anti-ADA antibody in 17 patients treated by intramuscular injections of PEG-ADA for 1 to $>5.5 \mathrm{yr}$. ELISA-detectable anti-ADA IgG appeared in 10 patients, usually between the third and eighth months of treatment. AntiADA levels did not correlate with trough plasma ADA activity, which averaged 1.8-5 times normal blood (erythrocyte) ADA activity, depending on dose (15-60 U/kg per wk). ELISA-detectable anti-ADA antibodies were directed primarily at bovine-specific peptide (rather than PEG-containing) epitopes. Enhanced enzyme clearance, mediated by antibody that directly inhibited native and PEG-modified bovine ADA, and native, but not PEG-modified human ADA, occurred in two patients. In one, tolerance was induced; in the second, twice weekly injections of PEG-ADA compensated for accelerated clearance. We speculate that inhibitory antibodies recognize conserved, relatively PEG-free epitope(s) encompassing the active site, and that in human, but not bovine, ADA a PEG-attachment site "shields" the active site from immune recognition. We conclude that PEG-modification largely prevents the development of high affinity, or high levels of, clearing antibodies to bovine ADA, and that PEG-modified human ADA should be further investigated as a possible treatment for ADA deficiency. (J. Clin. Invest. 1992. 89:1643-1651.) Key words: ELISA • inhibitory antibody • severe combined immunodeficiency disease • enzyme replacement therapy $\bullet$ immune tolerance
\end{abstract}

\section{Introduction}

The technique of modifying proteins by covalent addition of polyethylene glycol (PEG), aimed at slowing degradation and reducing immunogenicity, was developed to improve the characteristics of enzymes and other proteins for parenteral therapy (1-3). The approach has first been tested in the treatment of severe combined immunodeficiency disease (SCID) ${ }^{1}$ due to in-

Address correspondence and reprint requests to Michael S. Hershfield, M.D., Box 3049, Duke University Medical Center, Durham, NC 27710.

Received for publication 1 November 1991 and in revised form 30 December 1991.

1. Abbreviations used in this paper: ADA, adenosine deaminase; AdoHcyase, $S$-adenosylhomocysteine hydrolase; dAXP, total adenine deoxyribonucleotides; IVIg, intravenous Ig; PNP, purine nucleoside phosphorylase; SCID, severe combined immunodeficiency disease.

J. Clin. Invest.

(c) The American Society for Clinical Investigation, Inc.

0021-9738/92/05/1643/09 \$2.00

Volume 89, May 1992, 1643-1651 herited deficiency of adenosine deaminase (ADA) (4-6). Initial studies of patients treated for several months with PEG-modified bovine ADA (PEG-ADA, ADAGEN ${ }^{\mathrm{TM}}$ ) showed that weekly intramuscular injections produced high levels of ADA activity in plasma, corrected metabolic abnormalities, and led to improved immune function (6-9).

Successful replacement therapy of this usually fatal disorder requires continuous maintenance of high circulating levels of ADA activity, which could be jeopardized by the development of clearing antibodies to PEG-ADA. Before clinical trial, PEGylation had been shown to reduce both the antigenicity and the immunogenicity of bovine ADA in mice (10). Nevertheless, there were concerns that residual immunogenicity would limit the use of PEG-ADA in humans as chronic replacement therapy of ADA deficiency (11). Indeed, treatment with PEG-ADA entails significant, repeated antigenic challenge: 2.5 $\mathrm{ml}$ of the clinical preparation ADAGEN ${ }^{\mathrm{TM}}$, which several patients receive weekly, has the ADA activity of $\sim 10^{12}$ normal T cells or 4.5 liters of normal erythrocytes. Antibodies could arise towards "nonhuman" regions of PEG-ADA, either bovinespecific peptide segments or epitopes involving PEG. Conserved regions might also be immunogenic in patients who may never have developed tolerance to the native human ADA gene product: in addition to their immune deficit, most cell lines from ADA-deficient SCID patients have lacked or had very low levels of immunologically detectable ADA $(12,13)$. We have tried to address these issues and to define the evolution of IgG anti-ADA antibodies and their impact on therapy in 17 of the first 18 patients, who have been under treatment with PEG-ADA for 1.5 to $>5.5 \mathrm{yr}$.

\section{Methods}

PEG-modified bovine ADA (PEG-ADA, ADAGEN ${ }^{\mathrm{TM}}$ ) and disuccinyl-monomethoxyPEG, $M_{r} 5,000$ (14) were provided by Enzon, Inc. (South Plainfield, NJ). The PEG enzyme was supplied as a sterile solu-

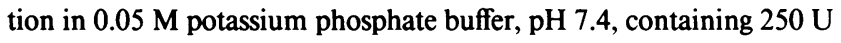
of ADA activity per ml measured at $25^{\circ}, \sim 550 \mathrm{U} / \mathrm{ml}$ measured at $37^{\circ}$ ( $1 \mathrm{U}=$ conversion of $1 \mu \mathrm{mol}$ of adenosine to inosine per min). Lots of PEG-ADA were changed according to the schedule of production. Except for brief periods, all patients were treated with the same lot of enzyme at any time.

Calf intestinal ADA was obtained from Sigma Chemical Co. (St. Louis, MO) and from Enzon. The preparation and characterization of PEG-modified Escherichia coli PEG-modified purine nucleoside phosphorylase (PNP) have been described (15). Purified recombinant human ADA was generously provided by Dr. Fred Rudolph, Rice University. It was treated with disuccinyl-monomethoxy PEG as described for preparation of PEG-PNP (15), resulting in modification of $40 \%$ of primary amino groups, as estimated by reaction with fluorescamine (16).

Enzyme assays and measurement of erythrocyte nucleotides. Fractionation of heparinized blood, radiochemical assays for plasma ADA and erythrocyte $S$-adenosylhomocysteine hydrolase (AdoHcyase) activities, and HPLC analysis of total erythrocyte adenine deoxyribonucleotides (dAXP) were performed as previously described and referenced in 
$(6,17)$. Protein was determined by the BCA method (Pierce Chemical Co., Rockford, IL).

Preparation of IgG from plasma. Plasma was diluted 1:1 with 0.15 $\mathrm{M} \mathrm{NaCl}, 0.01 \mathrm{M}$ potassium phosphate, $\mathrm{pH} 7.0,5 \mathrm{mM}$ EDTA (PBS/ EDTA) and centrifuged for $20 \mathrm{~min}$ in a microcentrifuge; then $0.45 \mathrm{ml}$ of supernatant was applied to a 1-ml column of Protein A Sepharose CL-4B (Sigma Chemical Co.) equilibrated with PBS/EDTA. After 30 min the column was washed with $21 \mathrm{ml}$ of PBS/EDTA (ADA activity eluted in this step). IgG was then eluted with $6 \mathrm{ml}$ of $0.1 \mathrm{M}$ sodium citrate, $\mathrm{pH}$ 3.0. After dialysis against $\mathrm{PBS}$, the IgG fraction was brought to $40 \%$ saturated ammonium sulfate and kept on ice for $1 \mathrm{~h}$; the precipitate was then collected by centrifugation, washed with $40 \%$ saturated ammonium sulfate, dissolved in $0.2-0.3 \mathrm{ml}$ of PBS, and dialyzed overnight against PBS. The final volume was brought to $0.45 \mathrm{ml}$ with PBS and stored at $-70^{\circ}$.

ELISA. Polystyrene 96-well microtiter plates (Immulon 2; Dynatech Laboratories Inc., Chantilly, VA) were treated overnight at $4^{\circ}$ with $0.1 \mathrm{ml}$ of purified, unmodified bovine ADA $(10 \mu \mathrm{g} / \mathrm{ml}$ in $0.1 \mathrm{M}$ sodium carbonate, pH 9.8), or with buffer alone (no-antigen control). After washing with PBS, wells were blocked with $0.2 \mathrm{ml}$ of $2 \%$ goat serum (Gibco BRL, Gaithersberg, MD) in PBS for $1 \mathrm{~h}$ at room temperature, then washed with PBS containing $0.1 \%$ Tween 20 . Patient plasma $(0.1$ $\mathrm{ml}$ ) sequentially diluted threefold from $1: 10$ to $1: 2,430$ in PBS, $2 \%$ goat serum, was added to antigen-coated and antigen-free wells in parallel rows. After $1 \mathrm{~h}$ at $37^{\circ}$ plates were washed with PBS containing $0.1 \%$ Tween 20 and bound human IgG was detected with a biotinylated goat anti-human IgG (gamma chain specific)-avidin-horseradish peroxidase detection system (Vectastain ABC kit; Vector Laboratories, Burlingame, CA), using $\sigma$-phenylenediamine as peroxidase substrate. After 25-30 min at room temperature the peroxidase reaction was stopped by addition of $0.1 \mathrm{ml} /$ well of $1 \mathrm{~N} \mathrm{HCl}$ and absorbance at $490 \mathrm{~nm}\left(\mathrm{~A}_{490}\right)$ was measured with a $V_{\max }$ Kinetic Microplate Reader (Molecular Devices Corp., Palo Alto, $\mathrm{CA}$ ). After subtracting values from no-antigen wells, $\mathrm{A}_{490}$ vs. $\mu$ l of plasma was plotted; the level of anti-ADA was estimated from the linear region of the plot and expressed as the theoret- ical $A_{490}$ per $10 \mu$ l of plasma. The same protocol was used in some experiments to detect antibody binding to immobilized PEG-ADA and to human ADA.

Competition ELISA. Aliquots $(0.05 \mathrm{ml})$ of a predetermined dilution of patient plasma (sufficient to give an absorbance of $0.5-1.5$ in the anti-ADA ELISA) were mixed with $0.05 \mathrm{ml}$ of $2 \%$ goat serum (control), or with $2 \%$ goat serum containing increasing amounts $(0.0016-20 \mu \mathrm{g})$ of unmodified or PEG-modified ADA, or PEG-modified $E$. coli PNP. After overnight incubation at $4^{\circ}$, these mixtures were tested in the ELISA as above (i.e., using unmodified bovine ADA as the immobilized antigen). A decrease in ELISA response relative to the control indicated binding of antibody by the antigen in solution.

Assay for ADA-inhibitory antibody. Duplicate aliquots (usually 10 $\mu$ l) of patient plasma or plasma IgG fraction (fraction B above), or an equal volume of $0.1 \%$ bovine serum albumin in PBS (PBS/BSA), were combined with PEG-ADA $\left(0.1-0.3 \mathrm{mU}\right.$ at $37^{\circ}$, prepared by dilution in PBS/BSA) and brought to $50 \mu$ l with PBS/BSA. After $30 \mathrm{~min}, 5 \mu 1$ aliquots were removed for radiochemical assay of ADA activity (final vol $50 \mu \mathrm{l}$ ) (17). Reactions performed without PEG-ADA were used to correct for ADA activity in the plasma or IgG fraction (the latter was always negligible). Percent inhibition of ADA activity was determined relative to activity obtained with PBS/BSA. The same procedure was used to measure inhibition of unmodified bovine ADA and human ADA (native and PEG-modified).

Patient characteristics. We evaluated anti-ADA antibody responses during 12-58 mo of treatment in 17 of the first 18 ADA-deficient SCID patients to receive PEG-ADA (designated by number according to the order in which they began treatment). Patient 15 , who has been under treatment for $16 \mathrm{mo}$, is not included because antibody was followed for only the first 6 mo of therapy (none was detected). Six patients began treatment at 3.8-14.9 yr of age, the other eleven at $6 \mathrm{wk}-26 \mathrm{mo}$ (Table I $A$ ). No patient had an HLA-matched sibling bone marrow donor. Before PEG-ADA, patient 1 had twice received HLA-haploidentical bone marrow transplants, but without stable engraftment (18); patients 2-4 had been treated for 9-10 yr with monthly (approximately) transfu-

Table I. Patient Profile (A) and ELISA Response to Unmodified Bovine ADA (B)

\begin{tabular}{|c|c|c|c|c|c|c|c|}
\hline \multirow[b]{2}{*}{$\begin{array}{l}\text { A. } \\
\text { Patient* } \\
\text { (sex) }\end{array}$} & \multirow[b]{2}{*}{$\begin{array}{l}\text { Age at start of } \\
\text { therapy }\end{array}$} & \multirow[b]{2}{*}{$\begin{array}{l}\text { Months on } \\
\text { of therapy }\end{array}$} & \multirow[b]{2}{*}{$\begin{array}{l}\text { Maintenance dose } \\
\text { of PEG-ADA }\end{array}$} & \multirow[b]{2}{*}{$\begin{array}{l}\text { B. } \\
\text { Month onset } \\
\quad+\text { ELISA }\end{array}$} & \multicolumn{3}{|c|}{ Anti-ADA ELISA ELISA response } \\
\hline & & & & & $\begin{array}{l}\text { Before } \\
\text { therapy }\end{array}$ & $\begin{array}{l}\text { During treatment } \\
\text { with PEG-ADA }\end{array}$ & \\
\hline & mo & & $U / k g$ per $w k$ & & $U$ & $U$ & mo \\
\hline $1(\mathrm{~F})$ & 45 & 67 & 20 & $5-6$ & 27.4 & $60.2 \pm 11(43-88)^{8}$ & $9-58^{\prime \prime}$ \\
\hline $2(\mathrm{~F})$ & 117 & 62 & 20 & - & 0.5 & $1.2 \pm 1 \quad(0-3)$ & $9-53^{\prime \prime}$ \\
\hline $3(\mathrm{M})$ & 144 & 57 & 20 & $5-6$ & 3.8 & $33.7 \pm 9 \quad(20-48)$ & $8-48^{\prime \prime}$ \\
\hline $4(\mathrm{M})$ & 115 & 56 & 15 & $6-8$ & 0.7 & $34.0 \pm 10(20-52)$ & $10-46^{\prime \prime}$ \\
\hline $5(F)$ & 69 & 55 & 20 & $1-2$ & 1.1 & $53.1 \pm 15(29-87)$ & $8-45^{\prime \prime}$ \\
\hline $6(\mathrm{M})$ & 1.6 & 52 & 30 & $24-28$ & 0.6 & $18.1 \pm 9 \quad(8-28)$ & $28-43^{\prime \prime}$ \\
\hline $7(\mathrm{M})$ & 22 & 39 & 20 & $4-6$ & 4.9 & $11.4 \pm 5 \quad(6-22)$ & $9-26^{11}$ \\
\hline $8(F)$ & 178 & 39 & 20 & $4-5$ & 2.8 & $54.8 \pm 16(36-87)$ & $10-29^{\prime \prime}$ \\
\hline $9(\mathrm{~F})$ & 26 & 36 & 25 & $2-4$ & 24.8 & $38.4 \pm 16(6-56)$ & $8-26^{11}$ \\
\hline $10(F)$ & 22 & 34 & 60 & - & 0.7 & $4.3 \pm 3 \quad(2-9)$ & $7-25^{\prime \prime}$ \\
\hline $11(\mathrm{M})$ & 1.7 & 34 & 60 & - & 0.2 & $2.4 \pm 2 \quad(0-5)$ & $8-24^{\prime \prime}$ \\
\hline $12(\mathrm{~F})$ & 16 & 29 & 60 & $4-5$ & 2.5 & $52.1 \pm 23(29-99)$ & $9-20^{\prime \prime}$ \\
\hline $13(\mathrm{M})$ & 19 & 21 & 30 & - & 0.3 & $2.2 \pm 1 \quad(1-4)$ & $6-17^{11}$ \\
\hline $14(\mathrm{~F})$ & 8 & 18 & 30 & $6-7$ & 3.6 & $7.8 \pm 5 \quad(2-17)$ & $6-14^{\prime \prime}$ \\
\hline $16(\mathrm{M})$ & 11 & 16 & 30 & - & 2.4 & $1.7 \pm 1 \quad(1-3)$ & $7-14^{\prime \prime}$ \\
\hline $17(\mathrm{M})$ & 2 & 13 & 30 & - & 1.9 & $3.5 \pm 2 \quad(0-5)$ & $7-12^{11}$ \\
\hline $18(\mathrm{M})$ & 6 & 12 & 30 & - & 2.2 & $3.8 \pm 1 \quad(3-5)$ & $6-12^{11}$ \\
\hline
\end{tabular}

* Numbered according to order of starting treatment with PEG-ADA; patient 15 is not included because complete data were not collected. ${ }^{\ddagger}$ As of November 1, 1991. " Mean \pm SD (range). "Treatment period (months) used for determining mean ELISA level. 
sions of irradiated erythrocytes, used as a form of enzyme replacement therapy (19); patients 1, 5, 7, and 10-12 had received transfusions for shorter periods. At the time PEG-ADA therapy was initiated all patients were lymphopenic and had decreased lymphocyte mitogen responses. Their clinical characteristics and responses to PEG-ADA therapy will be summarized elsewhere. From April, 1986 until its approval by the U.S. Food and Drug Administration in March, 1990, PEG-ADA therapy was experimental and was performed with informed parental consent and approval of Investigational Review Boards.

PEG-ADA administration. The intramuscular dose of PEG-ADA was adjusted to maintain preinjection (trough) plasma ADA activity at $>12 \mu \mathrm{mol} / \mathrm{h}$ per $\mathrm{ml}$ (approximately equivalent to total erythrocyte ADA activity in normal individuals), sufficient to lower red cell dAXP to $<10-15 \mathrm{nmol} / \mathrm{ml}$ packed cells and increase red cell AdoHcyase activity into the normal range (6-8). In most cases these conditions were achieved with one weekly injection of $15-30 \mathrm{U} / \mathrm{kg}$. Some newly diagnosed patients who were malnourished or severely ill initially required twice weekly injections of $30 \mathrm{U} / \mathrm{kg}$; this was changed to one injection a week when clinical status and $T$ cell function improved. The weekly maintenance doses that patients received during the period when average ELISA levels were determined (see Results) are listed in Table I $A$. The effect of higher doses on immune function is being evaluated in some patients, but here we will consider only the relationship of dose, plasma ADA activity, and anti-ADA antibody response.

\section{Results}

ELISA-detectable anti-ADA antibody. The standard ELISA measures IgG antibody to unmodified bovine ADA; specificity was established by the ability of antigens in solution to inhibit the ELISA. Pretreatment values for 15 patients averaged $2.11 \pm 1.6$ (SD) U (range 0.5-4.7) (Table I $B$ ), vs. $2.94 \pm 2.4 \mathrm{U}$ for 26 controls. Pretreatment levels of $25-30 U$ for patients 1 and 9 were nonspecific, i.e., inhibited neither by unmodified ADA

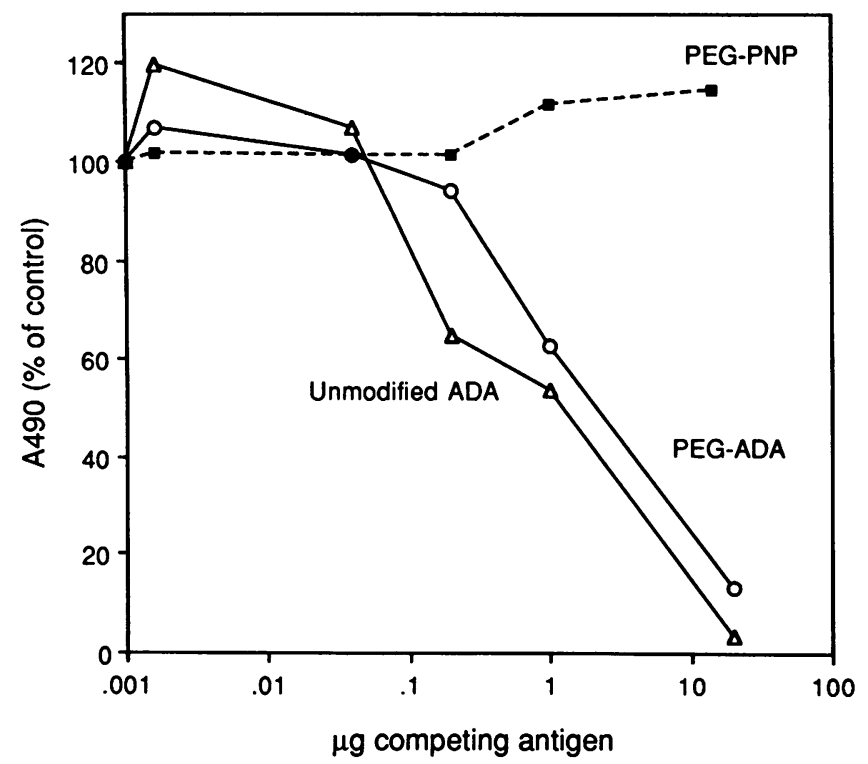

Figure 1. Specificity of anti-bovine ADA ELISA. Aliquots of diluted plasma (containing $0.37 \mu \mathrm{l}$ of plasma) obtained from patient 4 after 18 mo of therapy were mixed with PBS (control) or with the indicated amounts of unmodified bovine ADA (triangles), PEG-ADA (circles), or PEG-modified E. coli PNP (squares). As described in Methods, these mixtures were incubated overnight and then tested by ELISA for IgG antibody to unmodified bovine ADA.
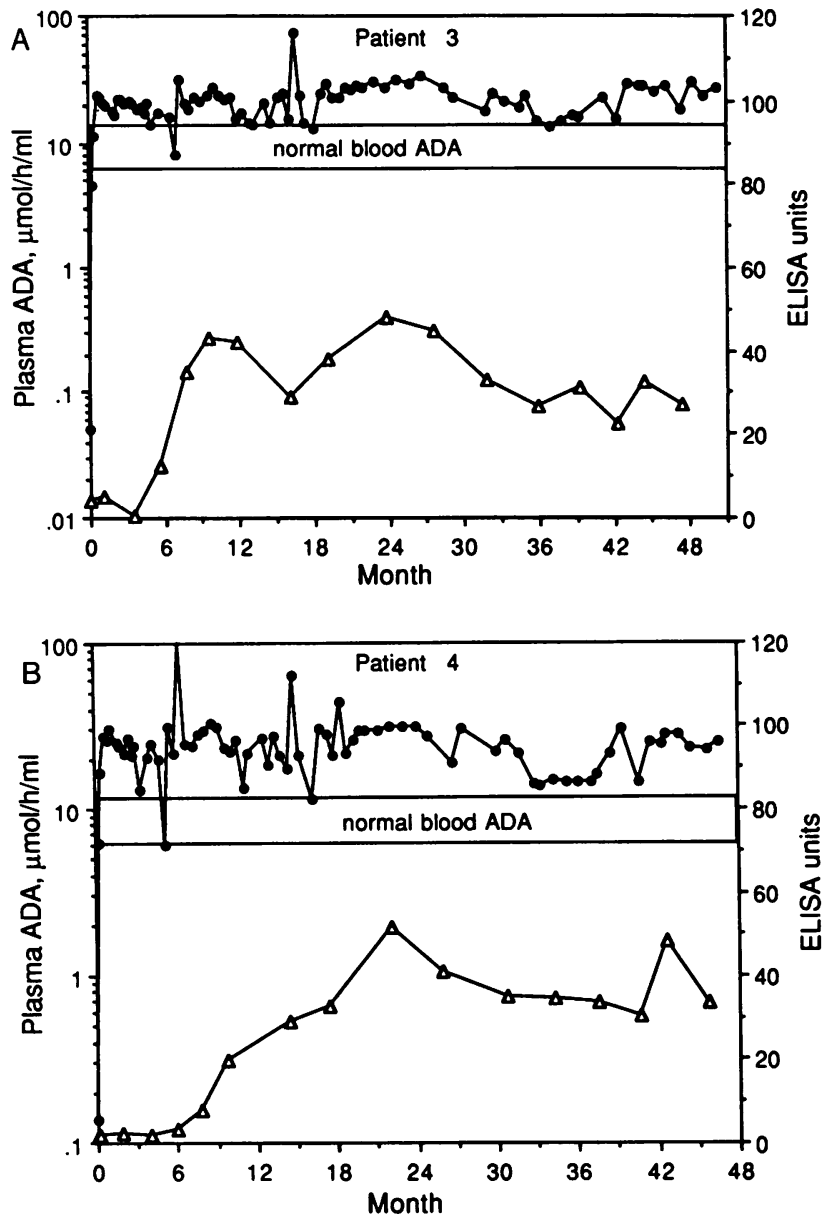

Figure 2. Evolution of anti-ADA antibody and relationship to trough (preinjection) plasma ADA activity. Filled circles, plasma ADA activity; open triangles, anti-ADA ELISA. Data are shown for patients $3(A)$ and $4(B)$.

nor by PEG-ADA (data not shown). As illustrated in Fig. 1 with plasma obtained from patient 4 after 18 mo of therapy, the specific ELISA response was inhibitable by preincubation with either unmodified bovine ADA or PEG-ADA, but not by PEG-modified purine nucleoside phosphorylase.

For each patient we determined a mean treatment ELISA value by testing 6-18 samples covering a 6-50-mo period beginning with the sixth to tenth month of therapy (Table I $B$ ). The mean ELISA value has remained negative $(<7.8$ units, 2 SD above the mean for controls) in 7 patients; 10 have developed specific anti-ADA IgG responses ranging from 8 to 55 ELISA U (average values). In most cases, the ELISA became positive between the third and eighth month of therapy (Table I $B)$ and remained positive thereafter, as illustrated for patients 3 and 4 (Fig. 2). There was no correlation between mean ELISA values and mean trough plasma ADA activity (determined from biweekly to bimonthly measurements over the period used to determine average ELISA response) (Fig. $3 \mathrm{~A}$ ). Mean plasma ADA activities ranged from 21.2 to $62.7 \mu \mathrm{mol} / \mathrm{h}$ per ml (mean $\pm \mathrm{SD}=29.6 \pm 12$ ), or $\sim 1.8-5$ times the normal level of total erythrocyte ADA activity, and correlated fairly well with the weekly dose of PEG-ADA (Fig. $3 B$ ).

Fig. 4 compares inhibition of the standard ELISA by un- 


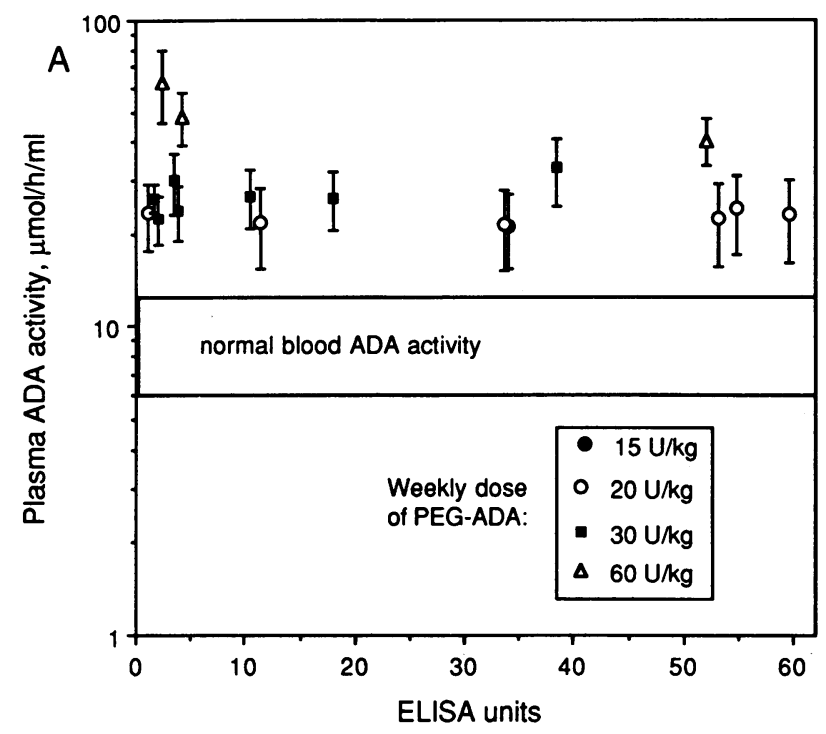

PEG-modified $E$. coli PNP at up to $200 \mu \mathrm{g} / \mathrm{ml}$, nor free PEG at up to $20 \mathrm{mg} / \mathrm{ml}$, inhibited the ELISA in any case (not shown).

In agreement with a preliminary report (20), ELISA responses were considerably lower when PEG-ADA was substituted for unmodified bovine ADA as immobilized antigen (each ELISA was performed at saturating antigen concentration). Values of 20-25 ELISA $U$ were obtained with plasma from patients 8 and $12,10 \mathrm{U}$ with patient 5 , and $0-6 \mathrm{U}$ with the other 7 patients (who all had significant responses to unmodified ADA). In the three patients with significant positive responses, the anti-PEG-ADA ELISA was inhibited equally by unmodified ADA and PEG-ADA (not shown). The insensitivity of the ELISA with immobilized PEG-ADA may reflect inaccessibility of some epitopes of the immobilized PEG enzyme to antibody.

$A D A$-inhibitory antibody. In 2 of the 17 patients (8 and 12), antibody to ADA did affect plasma ADA activity, requiring

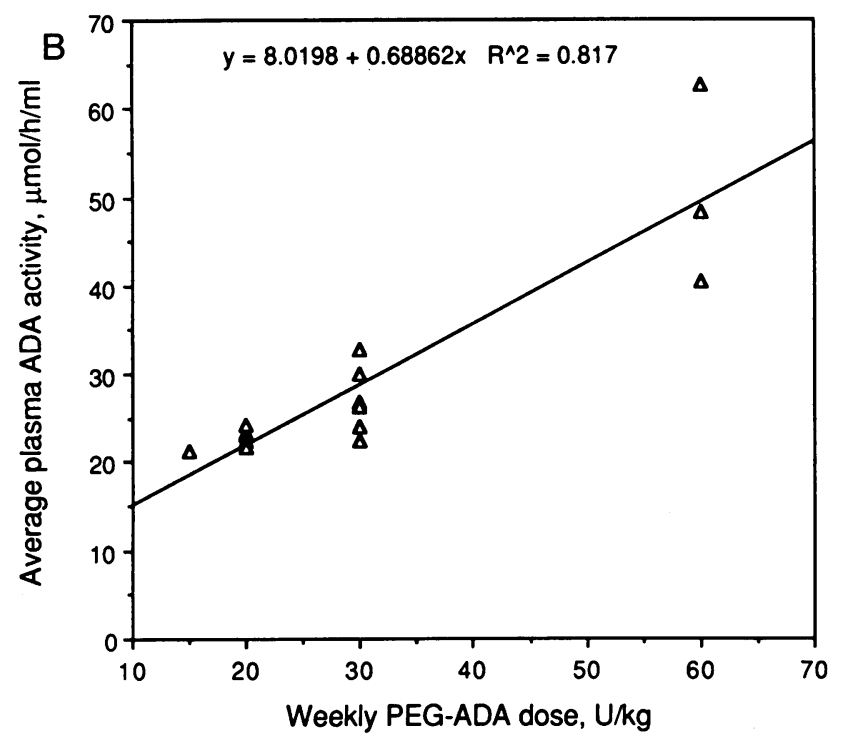

Figure 3. Relationships among anti-bovine ADA ELISA values, trough levels of plasma ADA activity, and weekly dose of PEG-ADA. $(A)$ The average treatment ELISA values for patients (horizontal axis) are plotted against their mean preinjection (trough) plasma ADA activity (vertical axis). Vertical bars attached to symbols are 1 SD; the different symbols represent the weekly dose of PEG-ADA, as indicated in figure. $(B)$ Weekly dose of PEG-ADA (horizontal axis) is plotted against average trough plasma ADA activity (vertical axis). The line was determined by least squares fit. The period of treatment evaluated is defined in Table I.

modified ADA and PEG-ADA in a study of recent plasma samples from seven anti-ADA positive patients. At $200 \mu \mathrm{g} / \mathrm{ml}$, each ADA species caused $80-100 \%$ inhibition. The concentration of competing antigen causing a 50\% decrease in ELISA response $\left(\mathrm{IC}_{50}\right)$ ranged from $\sim 2$ to $120 \mu \mathrm{g} / \mathrm{ml}\left(\sim 10^{3}\right.$ - to $10^{4}$ fold higher than the concentration of PEG-ADA present in the diluted patient plasma samples tested in the ELISA). The lowest $\mathrm{IC}_{50}$ occurred in patients 8 and 12 . In this experiment the $\mathrm{IC}_{50}$ for PEG-ADA was approximately twofold lower than for unmodified ADA, but no consistent difference between the two species was found in other similar experiments. Neither
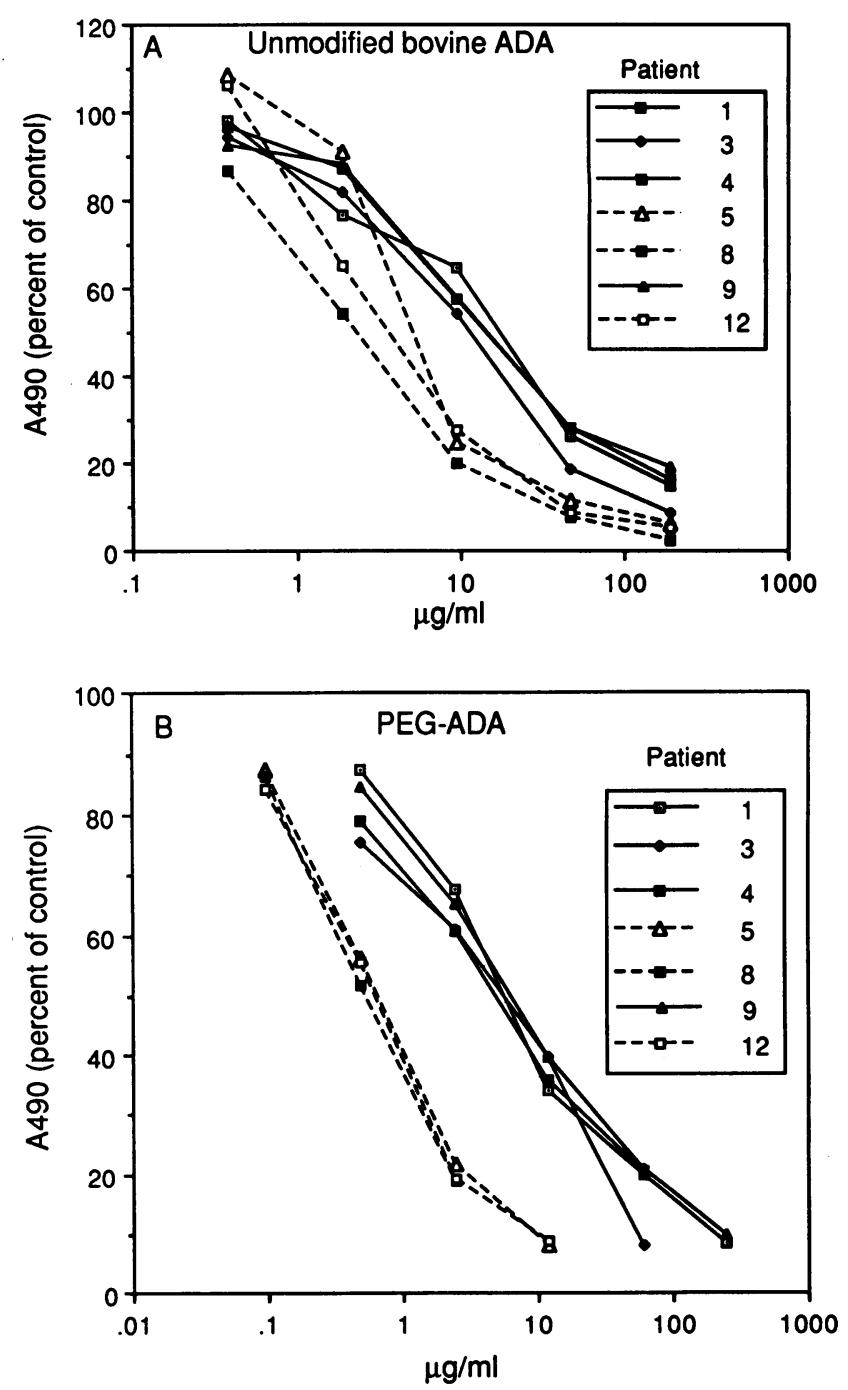

Figure 4. Inhibition of anti-bovine ADA ELISA. Diluted patient plasma samples were mixed with the indicated concentrations of unmodified bovine ADA $(A)$ or PEG-modified bovine ADA $(B)$, incubated overnight, and then tested by ELISA for IgG antibody to unmodified bovine ADA. The experimental protocol is described in Methods and in the legend to Fig. 1. 
treatment modification. We will review studies of patient 8 , which were aimed at defining and suppressing the effects of anti-ADA antibody. For the first 4 mo of therapy, a weekly PEG-ADA dose of $15 \mathrm{U} / \mathrm{kg}$ maintained trough plasma ADA activity at $13-23 \mu \mathrm{mol} / \mathrm{h}$ per $\mathrm{ml}$. Between weeks 21 and 25 the level fell to $<0.1 \mu \mathrm{mol} / \mathrm{h}$ per $\mathrm{ml}$, associated with development of a positive ELISA (Fig. 5), accelerated enzyme clearance (Fig. $6 A$ ), and an increase in red cell dAXP (Fig. $6 B$ ). Thus, during the first two weeks of treatment plasma ADA activity peaked $48-72 \mathrm{~h}$ after injections of 10 and $15 \mathrm{U} / \mathrm{kg}$, then declined with a half-life of $>6 \mathrm{~d}$. At week 26, following a dose of $20 \mathrm{U} / \mathrm{kg}$, plasma ADA peaked at $24 \mathrm{~h}$ at about half the level achieved during week 1 , then fell with a half-life of $<2 \mathrm{~d}$ to $0.1 \mu \mathrm{mol} / \mathrm{h}$ per $\mathrm{ml}$ by day 7 .

Plasma samples obtained from patient 8 between weeks 25 and 33, which had very low ADA activity, directly inhibited both PEG-ADA and unmodified bovine ADA; no inhibition was observed with pretreatment or control plasma (which in some cases stimulated slightly the activity of highly diluted ADA) (Table II). In mixing experiments week 26 plasma from patient 8 inhibited the ADA activity in plasma of other PEGADA-treated patients (data not shown). The ADA inhibitor was not dialyzable and could be recovered in the $\mathrm{IgG}$ fraction, separated from PEG-ADA, when the patient's plasma was fractionated on a protein A Sepharose column. Neither the preparation of IVIg that the patient was receiving, nor IgG prepared from the plasma of other ELISA-positive patients, appreciably inhibited ADA (Table II). The evolution of ADA inhibitory activity during the first 6 mo of treatment paralleled the development of ELISA-detectable anti-ADA IgG (Fig. 5).

In an attempt to suppress the effects of antibody (21), PEGADA was withheld between weeks 27 and 34, then resumed with three injections of $20 \mathrm{U} / \mathrm{kg}$ during week 35 , then continued at twice weekly injections of this dose for four months (Fig. 5). The patient also received high dose infusions of IVIg weekly

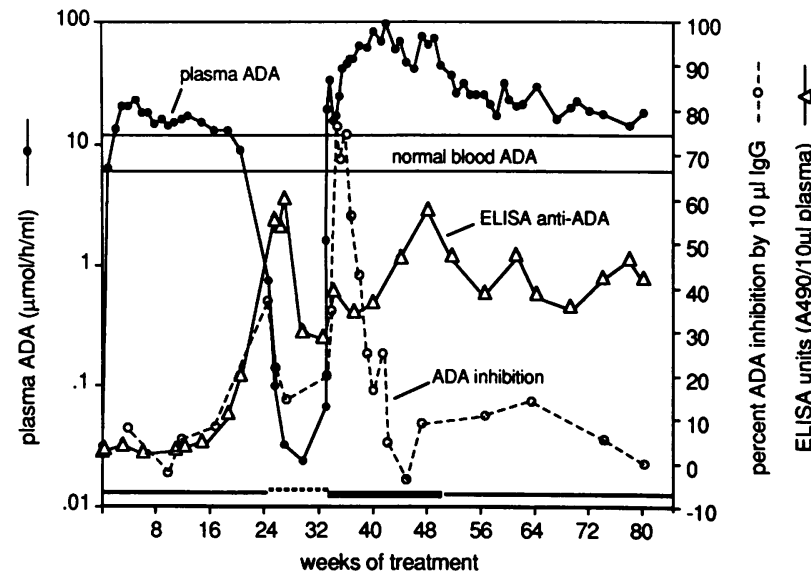

Figure 5. Evolution of anti-ADA antibody, effect on plasma ADA activity, and induction of tolerance to PEG-ADA in patient 8 . Plasma ADA levels (filled circles) were determined in preinjection samples. IgG antibody to unmodified bovine ADA was determined by ELISA (open triangles), and by ability of IgG isolated from patient plasma samples to inhibit PEG-ADA (open circles), as described in Methods. The horizontal lines above the $x$ axis indicate dose of PEG-ADA: thin line, one injection per week; dashed line, no injections; thick line, two injections per week.
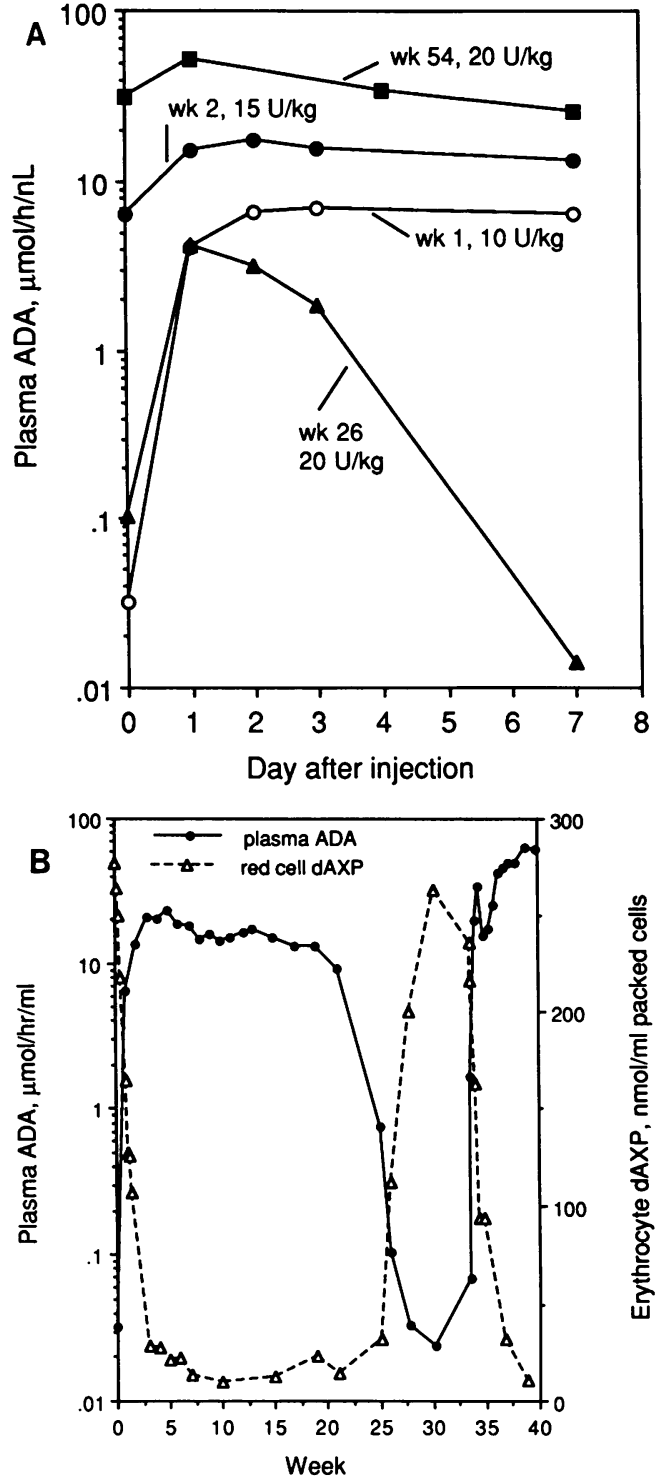

Figure 6. $(A)$ Clearance of PEG-ADA. Plasma ADA levels were determined following intramuscular injection of PEG-ADA; dose and week of treatment are indicated in the figure. $(B)$ Relationship between plasma ADA activity and erythrocyte dAXP in patient 8 .

for a month and a course of prednisone tapered over four months (this regimen and the patient's clinical response to PEG-ADA therapy will be described elsewhere). Six days after PEG-ADA was reinstituted, plasma ADA activity reached 34 $\mu \mathrm{mol} / \mathrm{h}$ per $\mathrm{ml}$ but then, despite the more frequent injection schedule, the ADA level fell to $15-17 \mu \mathrm{mol} / \mathrm{h}$ per $\mathrm{ml}$ on days 9-12. This coincided with a sharp, apparently anamnestic increase in IgG ADA-inhibitory activity, which was not reflected in the ELISA (Fig. 5, weeks 34-36). Over the next eight weeks ADA inhibitory antibody (but not ELISA-detectable antiADA) declined to the pretreatment baseline and plasma ADA activity increased to $41-96 \mu \mathrm{mol} / \mathrm{h}$ per $\mathrm{ml}$, resulting in depletion of red cell dAXP (Fig. 6 B). At week 52, PEG-ADA was returned to a single weekly dose of $20 \mathrm{U} / \mathrm{kg}$. The kinetics of PEG-ADA clearance examined during week 54 resembled the pattern observed at the beginning of treatment (Fig. $6 \mathrm{~A}$ ). Trough plasma ADA activity has ranged from 14 to $39 \mu \mathrm{mol} / \mathrm{h}$ 
Table II. Inhibition of PEG-ADA Activity by Anti-ADA Antibody from Patient 8

\begin{tabular}{llcc}
\hline $\begin{array}{c}\text { Sample } \\
\text { type }\end{array}$ & \multicolumn{1}{c}{ Source } & $\begin{array}{c}\text { Week(s) of } \\
\text { treatment }\end{array}$ & $\begin{array}{c}\text { Percent inhibition } \\
\text { of PEG-ADA activity }\end{array}$ \\
\hline Plasma & Controls $(n=4)$ & - & $-14.4 \pm 11.7^{*}$ \\
& & & $(0.4 \text { to }-27.9)^{\ddagger}$ \\
Plasma & Patient 8 & Pretreatment & -15.5 \\
Plasma & Patient 8 & 25 & 28.4 \\
Plasma & Patient 8 & 27 & 79.4 \\
IgG & Pooled IVIg & - & 2.8 \\
IgG & Control & - & 1.1 \\
IgG & Patient 8 & Pretreatment & -1.6 \\
IgG & Patient 8 & $4-17$ & $2.8 \pm 3.6$ \\
IgG & Patient 8 & 25 & 22.1 \\
IgG & Patient 8 & 36 & 75.9 \\
IgG & Other ELISA & $20-141$ & $4.5 \pm 5.1$ \\
& + patients & \\
& & & $(-2.2$ to 11.2$)$
\end{tabular}

${ }^{*}$ mean $\pm \mathrm{SD} ;{ }^{\ddagger}$ (range). ${ }^{\S}$ Patients $1,3,4,5$, and 6.

per $\mathrm{ml}$ for the $>24$ mo since week 55, even though anti-ADA antibody detectable by ELISA has persisted at levels $>50 \mathrm{U}$. Maintenance of adequate ADA levels has been accompanied by improvement in immune function and sustained freedom from serious infections (J. D. Chum et al., manuscript in preparation).

In the experiment shown in Fig. 7, inhibition of ADA activity was measured when increasing amounts of PEG-ADA were incubated with a fixed amount of ADA-inhibitory IgG. The units (in moles) of ADA inhibited (i.e., bound by inhibitory antibody) increased in a saturable manner as a function of ADA concentration (Fig. $7 \mathrm{~A}$ ). A Scatchard plot of the ratio of inhibited (bound)-to-active (free) enzyme vs. inhibited (bound) enzyme gave a straight line (Fig. $7 \mathrm{~B}$ ), indicating a single species of inhibitory antibody. From this plot we estimate a $K_{d}$ of $\sim 0.5 \mathrm{nM}$ for the antibody-PEG-ADA complex (this is similar to an approach used by Ehle et al. [22] to estimate the affinity of enzyme-inhibitory antibodies). Similar studies with unmodified bovine ADA gave an estimated $K_{d}$ of $\sim 0.7 \mathrm{nM}$ (data not shown).

Patient 12 also experienced a fall in plasma ADA after about four months of therapy, which coincided with appearance of a positive anti-ADA IgG ELISA (Fig. 8) and plasma ADA inhibitory activity (see below). PEG-ADA was stopped for two weeks, then restarted at $30 \mathrm{U} / \mathrm{kg}$ twice weekly, along with two high dose IVIg infusions and a tapering course of prednisone. From months 7 to 14, two weekly PEG-ADA injections of $25 \mathrm{U} / \mathrm{kg}$ maintained plasma ADA activity at $25.1 \pm 7.3 \mu \mathrm{mol} / \mathrm{h}$ per ml. When a once weekly injection schedule $(30 \mathrm{U} / \mathrm{kg})$ was resumed at month 14 , plasma ADA activity declined to 3-4 $\mu \mathrm{mol} / \mathrm{h}$ per $\mathrm{ml}$ and red cell dAXP increased from 6 to 12 to $\sim 50 \mathrm{nmol} / \mathrm{ml} \mathrm{packed} \mathrm{cells} \mathrm{(Fig.} \mathrm{8).} \mathrm{Twice}$ weekly injections of PEG-ADA (30 U/kg) were resumed, which has maintained plasma ADA activity between 30 and 50 $\mu \mathrm{mol} / \mathrm{h}$ per $\mathrm{ml}$. As reported elsewhere (23), each decline in plasma ADA activity was accompanied by a fall in red cell AdoHcyase activity and blood lymphocyte count, which reversed with increase in PEG-ADA dose. Patient 12 remains clinically well on continuing therapy with PEG-ADA.
Species specificity of anti-ADA antibodies. We have recently examined the ability of patient anti-ADA antibodies to recognize recombinant human ADA and its PEG-modified derivative. Plasma from 13 patients showed insignificant reaction with unmodified human ADA (ranging from undetectable to $5.8 \mathrm{U}$ ) when it was substituted for bovine ADA in the ELISA; patient 6 had a positive response of $11.8 \mathrm{U}$, vs. $21 \mathrm{U}$ with bovine ADA (Fig. $9 \mathrm{~A}$ ). When preincubated with patient plasma at $200 \mu \mathrm{g} / \mathrm{ml}$, unmodified and PEG-modified human ADA failed to inhibit or showed weak inhibition of a standard anti-bovine ADA ELISA compared with their bovine counterparts (data not presented). In contrast to the ELISA results, plasma samples from patients 8 and 12 inhibited the activity of unmodified human ADA, the former to the same degree and the latter $\sim 50 \%$ of the inhibition of bovine ADA and PEGADA preparations (Fig. 9 B). An important finding was that neither patient plasma inhibited PEG-modified recombinant human ADA.

\section{Discussion}

Specific IgG antibody to ADA reached significant levels ( $>2$ SD above the mean for controls in the standard ELISA) in 10 of the 17 patients studied, usually between three and eight months of treatment. This time course parallels improvement of impaired immune function, although some patients who have developed specific cellular and humoral responses to other antigens have not developed anti-ADA antibody (a separate report will deal with immunologic and clinical responses to treatment). Anti-ADA ELISA levels did not correlate with trough (preinjection) plasma ADA activity and, except in two patients, enzyme levels have been stable in anti-ADA positive patients over periods ranging from 24 to 60 months. We suspect that stable clearance reflects low affinity or low absolute levels of the population of antibodies generated towards epitopes incompletely masked by PEG strands (see below).

In two patients appearance of anti-ADA IgG was accompanied by a striking fall in plasma ADA activity, enhanced enzyme clearance, and the appearance in plasma of an IgG inhibitor of both PEG-ADA and unmodified ADA. An empirical approach (21) (details to be described elsewhere) was successful in inducing apparent tolerance to PEG-ADA in patient 8: PEG-ADA was withheld for several weeks, during which metabolic abnormalities and immune deficiency recurred; twiceweekly injections of PEG-ADA were then instituted, along with high dose IVIg and a tapering course of prednisone. ELISA-detectable anti-ADA IgG persisted, but after an abrupt anamnestic rise in level, ADA-inhibitory IgG disappeared over approximately two months and has not reappeared. Prolonged circulating life of PEG-ADA was restored, allowing treatment with a single weekly dose to be resumed. A similar regimen did not fully suppress the effects of antibody in patient 12 , but a twice-weekly injection schedule compensated for enhanced ADA clearance. Patients 8 and 12 have now received PEGADA for $>3 \mathrm{yr}$ and $>2.5 \mathrm{yr}$, respectively, and each has done well clinically (reference 23 and J. D. Chum et al., manuscript in preparation).

The reciprocal relationship between plasma ADA levels and ADA-inhibitory IgG in patient 8 indicates that the latter was responsible for enhanced enzyme clearance. Scatchard 

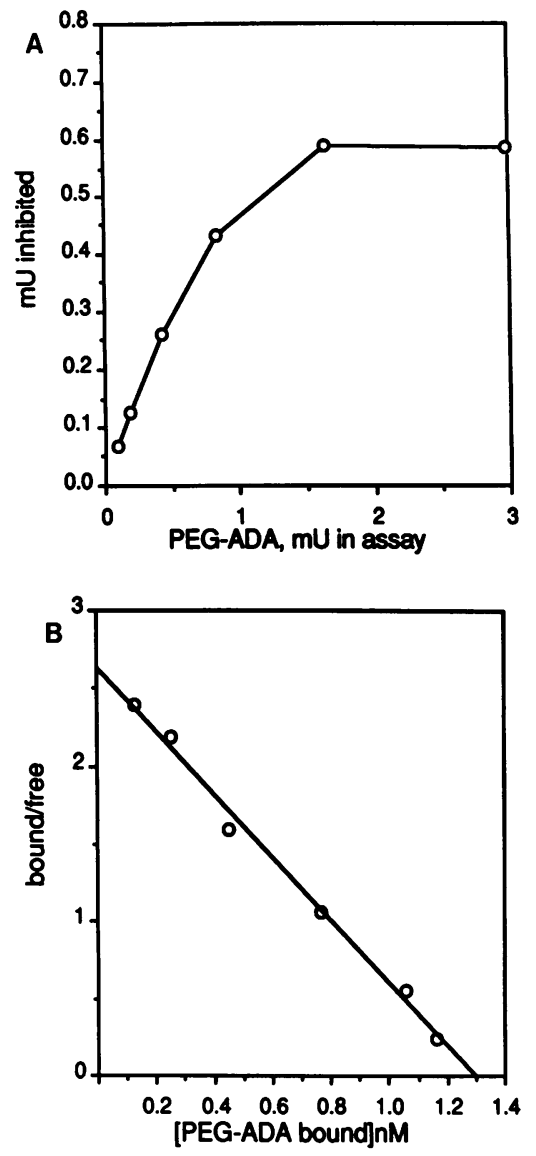

Figure 7. Relationship between ADA concentration and inhibition of $\mathrm{ADA}$ activity by IgG from patient 8 . Aliquots $(40 \mu \mathrm{l})$ of dilutions of PEG-ADA, prepared in PBS/BSA, were mixed with $10 \mu$ l of IgG prepared from plasma obtained at week 36 of treatment, or with $10 \mu \mathrm{l}$ of PBS/BSA (no-antibody control). After 20 min, $5 \mu$ aliquots were assayed for ADA activity. $(A)$ Relationship between ADA activity present in the assay and units of ADA activity inhibited (difference between activity found in the absence and presence of antibody). The percentage of activity inhibited ranged from 19.8 to $73 \%$. (B) Scatchard analysis of the data in $A$. The concentration of ADA present in the assay and the amounts bound (inhibited) and free (uninhib-

ited) were calculated from the measured specific activity and the concentration of ADA protein.

analysis of data relating ADA concentration to activity inhibition by $\mathrm{IgG}$ isolated from patient 8 indicated a single species of ADA-inhibitory antibody, possibly the product of one B cell

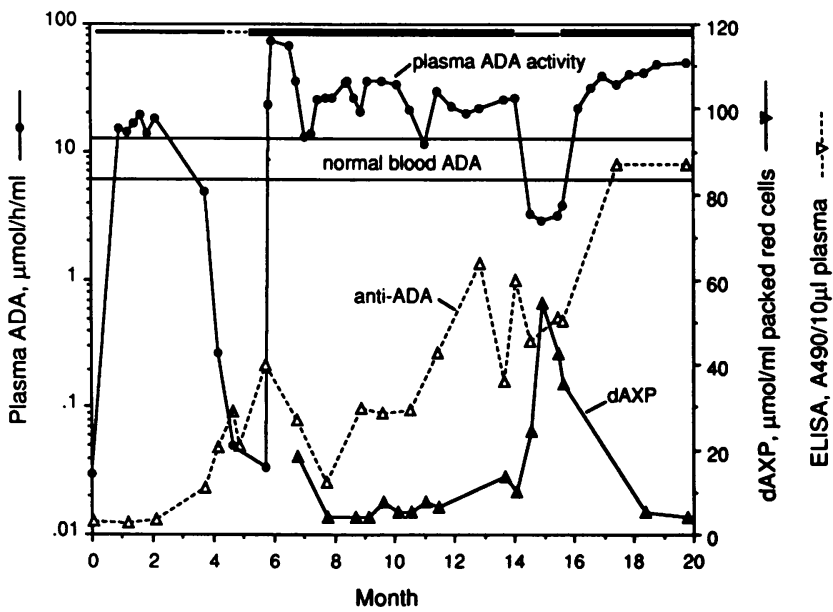

Figure 8. Anti-ADA antibody, plasma ADA activity, and red cell dAXP in patient 12. The horizontal lines at the top of the figure indicate the number of ADA injections per week: single thickness, one injection; dashed, no injections; double thickness, two injections. Data on red cell dAXP are shown for the period when the schedule of PEG-ADA administration was changed from two to one per week, and then returned to twice weekly.
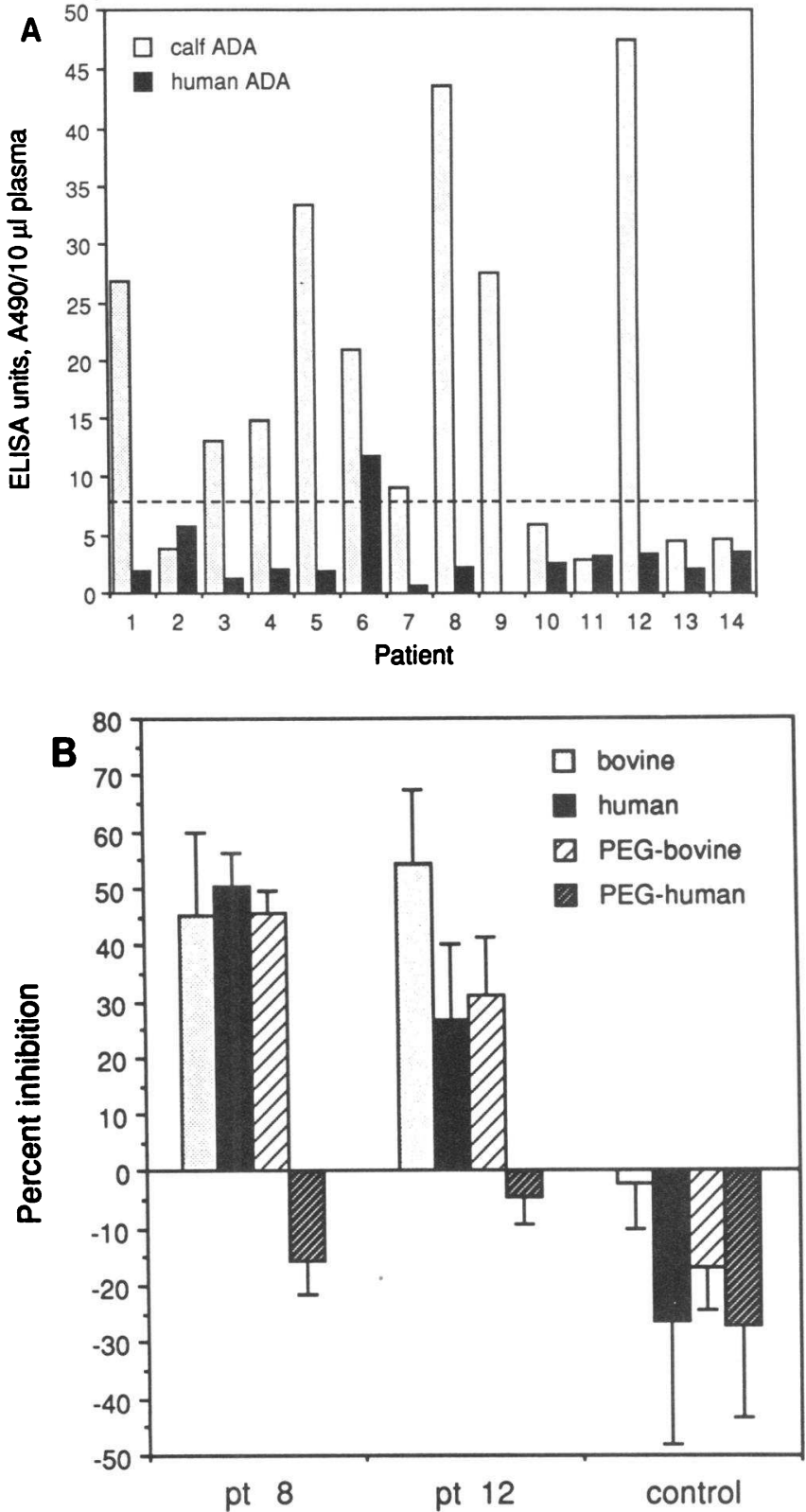

Figure 9. Comparison and antibody recognition of human and bovine ADA. (A) ELISA performed with unmodified calf (light bars) or human (dark bars) ADA as immobilized antigen. (B) Inhibition of bovine and human native ADA and PEG-ADA by plasma from patients 8 and 12. Plasma samples were from weeks 27 (patient 8 ) and 20 (patient 12) of therapy. Control is a plasma sample from a normal adult; the stimulation (negative inhibition) of purified ADA diluted in PBS/BSA was also observed with pretreatment plasma samples from patients and with plasma from other normal individuals (see Table II).

clone. The basis for the selective arrest in production of inhibitory antibody in this patient is unknown, but may be related to her underlying disorder as well as to properties of PEGylated antigens. In addition to the effects of prednisone and IVIg, immunosuppressive effects of metabolic abnormalities that recurred when ADA levels declined may have played a role in aborting the evolving inhibitory antibody response. PEGmodified allergens were toleragenic in mice, apparently by a 
mechanism that involved $\mathrm{T}$ cell suppression (24). On the other hand, the response in patient 8 resembles the experimental abrogation of primary immunization by high doses of soluble antigen, which may have resulted from direct effects on B cells (25). Although the use of steroids in a patient with SCID should be carried out with caution, the tolerizing regimen used in the present patients was much less intensive than that employed in hemophilia patients with high levels of inhibitory antibodies to Factor VIII:C, which included cyclophosphamide and plasmapheresis or protein A adsorption to remove circulating antibody (26).

Certain conclusions can be drawn regarding the specificity of anti-ADA IgG antibodies. (a) Protein epitopes rather than PEG are recognized: in competition experiments, native and PEG-modified bovine ADA were equally effective in blocking ELISAs performed with either species as the immobilized antigen; in contrast, neither a nonspecific PEG-protein nor free PEG had any effect. (b) Most anti-ADA antibodies recognize bovine-specific rather than conserved epitopes: ELISA responses to immobilized human ADA were low, and human ADA (native or PEG-modified) blocked the anti-bovine ADA ELISA weakly. $(c)$ In contrast to ELISA-detectable antibodies, the inhibitory antibodies of patients 8 and 12 recognize conserved region(s), since they inhibited human as well as bovine ADA. Both of these patients have detectable residual ADA activity so that development of inhibitory antibody is not related to a complete absence of the human ADA gene product. Rather, the active site of the PEG-modified enzyme may be a favorable site for immune recognition and antibody binding.

To preserve activity, PEG strands, which are linked to lysines, cannot be attached to an essential residue or block the active site. Consequently, a relatively PEG-free region around the active site may be an immunologic "Achilles heel." It is significant that while plasma from patients 8 and 12 inhibited native human ADA and native and PEG-modified bovine ADA, PEG-modified human ADA was not inhibited. We postulate that in human (but not bovine) ADA, a site for PEG attachment exists within the active site-related epitope, effectively shielding it from antibody. The postulated PEG-attachment site might also prevent an inhibitory antibody response to human PEG-ADA. The underlying hypothesis, that antibodies to PEG enzymes are generated against and bind to relatively PEG-free regions, is supported by recent studies in mice. We showed that the immunogenicity and antigenicity of PEGmodified wild type purine nucleoside phosphorylase from $E$. coli could be reduced substantially by using a mutant form of the enzyme in which arginine-to-lysine substitutions had been selectively introduced to provide additional sites for PEG attachment (15). We are currently investigating the epitope recognized by inhibitory anti-ADA antibody and we are pursuing the possible use of PEG-modified human ADA as a treatment for ADA deficiency.

Treatment with PEG-ADA has been a safe and effective way of maintaining the high levels of circulating ADA activity required to prevent toxic metabolic abnormalities caused by ADA deficiency. In more than five years of use (now in 25 patients) there have been no allergic reactions to PEG-ADA and in no patient has PEG-ADA been discontinued because of immunogenicity. Enhanced enzyme clearance, mediated by antibody, has occurred in two patients. However, it has been relatively easy to induce tolerance or to compensate for acceler- ated clearance by increasing the frequency of injections. Based on the present and ongoing studies, we recommend monitoring of preinjection plasma ADA levels every other week during the first six to eight months of therapy with PEG-ADA, then monthly; assay for anti-ADA antibodies by ELISA and by enzyme inhibition should be performed promptly if a persistent decline in enzyme levels occurs so that measures can be taken to provide appropriate prophylaxis against infection and to restore adequate enzyme levels.

\section{Acknowledgments}

Scott Muir, Christine Hatem, and Alison Brown provided expert technical assistance. We are grateful to Drs. M. Ballow, M. Berger, C. Bordignon, C. Bory, R. Buckley, S. Douglas, R. Kobayashi, A. Kobayashi, A. Rubenstein, R. Sorensen, G. Souillet, and K. Weinberg for providing blood samples from patients.

This research was supported by National Institutes of Health grant DK20902 (to M. S. Hershfield), and by Enzon, Inc.

\section{References}

1. Abuchowski, A., J. R. McCoy, N. C. Palczuk, T. van Es, and F. F. Davis. 1977. Effect of attachment of polyethylene glycol on immunogenicity and circulating life of bovine liver catalase. J. Biol. Chem. 252:3582-3586.

2. Abuchowski, A., T. van Es, N. C. Palczuk, and F. F. Davis. 1977. Alteration of immunological properties of bovine serum albumin by covalent attachment of polyethylene glycol. J. Biol. Chem. 252:3578-3581.

3. Abuchowski, A., and F. F. Davis. 1981. Soluble polymer-enzyme adducts In Enzymes as Drugs. J. S. Holcenberg and J. Robert, editors. John Wiley \& Sons, Inc., New York. 367-383.

4. Giblett, E. R., J. E. Anderson, F. Cohen, B. Pollara, and H. J. Meuwissen. 1972. Adenosine deaminase deficiency in two patients with severely impaired cellular immunity. Lancet. ii:1067-1069.

5. Kredich, N. M., and M. S. Hershfield. 1989. Immunodeficiency diseases caused by adenosine deaminase deficiency and purine nucleoside phosphorylase deficiency. In The Metabolic Basis of Inherited Disease. C. R. Scriver, A. L. Beaudet, W. S. Sly, and D. Valle, editors. McGraw-Hill Inc., New York. 10451075.

6. Hershfield, M. S., R. H. Buckley, M. L. Greenberg, A. L. Melton, R. Schiff, C. Hatem, J. Kurtzberg, M. L. Markert, R. H. Kobayashi, A. L. Kobayashi, and A. Abuchowski. 1987. Treatment of adenosine deaminase deficiency with polyethylene glycol-modified adenosine deaminase. N. Engl. J. Med. 316:589-596.

7. Levy, Y., M. S. Hershfield, C. Fernandez-Mejia, S. H. Polmar, D. Scudiery, M. Berger, and R. U. Sorensen. 1988. Adenosine deaminase deficiency with late onset of recurrent infections: response to treatment with polyethylene glycolmodified adenosine deaminase (PEG-ADA). J. Pediatr. 113:312-317.

8. Bory, C., R. Boulieu, G. Souillet, C. Chantin, M. O. Rolland, M. Mathieu, and M. S. Hershfield. 1990. Comparison of red cell transfusion and polyethylene glycol-modified adenosine deaminase therapy in an adenosine deaminase-deficiency child. Pediatr. Res. 28:127-130.

9. Hershfield, M. S., and S. Chaffee. 1991. PEG-Enzyme replacement therapy in adenosine deaminase deficiency. In Treatment of Genetic Diseases. R. J. Desnick, editor. Churchill-Livingstone Inc., New York. 169-182.

10. Davis, S., A. Abuchowski, Y. K. Park, and F. F. Davis. 1981. Alteration of the circulating life and antigenic properties of bovine adenosine deaminase in mice by attachment of polyethylene glycol. Clin. Exp. Immunol. 46:649-652.

11. Beauchamp, C., P. Daddona, and D. P. Menapace. 1984. Properties of a novel PEG derivative of calf adenosine deaminase. Adv. Exp. Med. Biol. 165B:47-52.

12. Wiginton, D. A., and J. J. Hutton. 1982. Immunoreactive protein in adenosine deaminase deficient human lymphoblast cell lines. J. Biol. Chem. 257:3211-3217.

13. Daddona, P. E., B. S. Mitchell, H. J. Meuwissen, B. L. Davidson, J. M. Wilson, and C. A. Koller. 1983. Adenosine deaminase deficiency with normal immune function. J. Clin. Invest. 72:483-492.

14 Abuchowski, A., G. M. Kazo, C. R. Verhoest, Jr., T. Van Es, D. Kaskewitz, M. L. Nucci, A. T. Viau, and S. S. Davis. 1984. Cancer therapy with modified enzymes. I. Antitumor properties of polyethylene glycol-asparaginase conjugates. Cancer Biochem. Biophys. 7:175-186.

15. Hershfield, M. S., S. Chaffee, L. Koro-Johnson, A. Mary, A. A. Smith, and S. A. Short. 1991. Use of site directed mutagenesis to enhance the epitope shield- 
ing effect of covalent modification of proteins with polyethylene glycol. Proc. Natl. Acad. Sci. USA. 88:7185-7189.

16. Stocks, S. J., A. J. M. Jones, C. W. Ramey, and D. E. Brooks. 1986. A fluorometric assay of the degree of modification of protein primary amines with polyethylene glycol. Anal. Biochem. 154:232-234.

17. Arredondo-Vega, F. X., J. Kurtzberg, S. Chaffee, I. Santisteban, E. Reisner, M. S. Povey, and M. S. Hershfield. 1990. Paradoxical expression of adenosine deaminase in $\mathrm{T}$ cells cultured from a patient with adenosine deaminase deficiency and combined immunodeficiency. J. Clin. Invest. 86:444-452.

18. Markert, M. L., M. S. Hershfield, R. Schiff, and R. H. Buckley. 1987 Adenosine deaminase and purine nucleoside phosphorylase deficiencies: evaluation of therapeutic interventions in eight patients. J. Clin. Immunol. 7:389-399.

19. Polmar, S. H., R. C. Stern, A. L. Schwartz, E. M. Wetzler, P. A. Chase, and R. Hirschhorn. 1976. Enzyme replacement therapy for adenosine deaminase deficiency and severe combined immunodeficiency. $N$. Engl. J. Med. 295:13371343.

20. Nucci, M. L., J. Olejarczyk, R. Buckley, M. Hershfield, A. Rubenstein, R Kobayashi, A. L. Kobayashi, R. Sorensen, and A. Abuchowski. 1988. Immunogenicity of polyethylene glycol-modified adenosine deaminase. J. Allergy Clin. Im munol. 81:276.

21. Lee, N., R. H. Kobayashi, S. Chaffee, M. S. Hershfield, and E. R. Stiehm.
1990. Suppression of an inhibitory antibody to bovine adenosine-deaminase (ADA) and improved cellular immunity following intravenous immunoglobulin and polyethylene-glycol (PEG-ADA). Pediatr. Res. 27:155A. (Abstr.)

22. Ehle, H., C. Godicke, and A. Horn. 1989. A new graphical method for determining the affinity constants of monoclonal antibodies to enzymes. $\mathrm{J}$. Im munol. Methods 17:17-23.

23. Girault, D., F. Le Deist, M. Debré, J. L. Pérignon, C. Herbelin, C. Griscelli, D. Scudiery, M. Hershfield, and A. Fischer. 1992. Traitement du deficit en adenosine desaminase par l'adenosine desaminase couplee au polyethylene glycol (PEG-ADA). Arch. Fr. Pediatr. In press.

24. Lee, W. Y., A. H. Sehon, and E. Akerblom. 1981. Suppression of reaginic antibodies with modified allergens. IV. Induction of suppressor T cells by conjugates of polyethylene glycol (PEG) and monomethoxy PEG with ovalbumin. Int Arch. Allergy Appl. Immunol. 64:100-114.

25. Nossal, G. J. V., and M. Karvelas. 1990. Soluble antigen abrogates the appearance of anti-protein IgG1-forming cell precursors during primary immunization. Proc. Natl. Acad. Sci. USA. 87:1615-1619.

26. Nilsson, I. M., E. Bernotorp, and O. Zettervall. 1988. Induction of immune tolerance in patients with hemophilia and antibodies to factor VIII by combined treatment with intravenous IgG, cyclophosphamide, and factor VIII. N. Engl. J. Med. 318:947-950. 